\title{
Immune checkpoint inhibitors and the shared epitope theory: from hypothesis to practice
}

\author{
Shalom Lerrer, Adam Mor \\ Columbia Center for Translational Immunology, Columbia University Medical Center, New York, NY, USA \\ Correspondence to: Adam Mor, MD, PhD. Columbia University Medical Center, 630 W 168 St. PH8-406, New York, NY 10032, USA. \\ Email: am5121@CUMC.Columbia.edu. \\ Comment on: Berner F, Bomze D, Diem S, et al. Association of Checkpoint Inhibitor-Induced Toxic Effects With Shared Cancer and Tissue Antigens \\ in Non-Small Cell Lung Cancer. JAMA Oncol 2019. [Epub ahead of print].
}

Submitted Jul 02, 2019. Accepted for publication Jul 23, 2019.

doi: $10.21037 /$ tcr.2019.07.43

View this article at: http://dx.doi.org/10.21037/tcr.2019.07.43

Checkpoints are inhibitory receptors expressed on activated $T$ cells. During the process of carcinogenesis, tumor cells progressively express multiple inhibitory receptor-ligands to prevent $T$ cell recognition and elimination. Consequently, therapeutic blockade of these checkpoints, or their ligands, helps recover anti-tumor immunity. Checkpoint inhibitors have been considered as a new armory for cancer patients due to the broad effectiveness of three agents blocking the inhibitory receptors CTLA-4, PD-1, and PD-L1 (the ligand for PD-1) (1). Based upon prolonged overall survival in clinical trials, antibodies inhibiting CTLA4, PD-1 and PD-L1 have been approved by the FDA for multiple clinical indications, including melanoma, nonsmall cell lung cancer (NSCLC), head and neck squamous cell carcinoma, urothelial carcinoma, renal cell carcinoma, Hodgkin lymphoma, and other adult and pediatric solid tumors.

The PD-1-PD-L1 interaction inhibits not just antitumor $\mathrm{T}$ cells, but also promotes peripheral effector $\mathrm{T}$ cells exhaustion, and supports the conversion of effector $\mathrm{T}$ cells into suppressive regulatory $\mathrm{T}$ cells (2). Accordingly, one in every four patients treated with antibodies targeting checkpoints will develop a unique spectrum of immunerelated adverse events (irAEs) (3). These events are associated with significant morbidities and in some cases life-long disability. Moreover, some patients require longterm immune suppressive treatment and others have to withdraw from lifesaving anti-cancer therapy. As such, identifying the mechanisms of irAEs development is of utmost clinical importance. While the molecular pathways triggering irAEs are largely unknown, it is likely that some are elicited by self-reactive $\mathrm{T}$ cells, which facilitate tissuespecific inflammation and provide B cell help for subsequent auto-antibody production (4).

In an elegant work published this year in JAMA Oncology, Berner et al., aimed to uncover the mechanism of irAEs among NSCLC patients treated with anti-PD-1 antibodies (5). First, they reported an association between site of irAEs involvement with shared cancer and tissue known antigens. Interestingly, skin was the second most similar organ to NSCLC tissue (after lung). Through a prospective cohort of 73 NSCLC patients receiving anti-PD-1 treatment, they identified 25 patients that developed skin-irAEs. Confirming previous observations, and as expected, irAEs, including skin toxicity, were more frequent in patients that responded favorably to anti-PD-1 treatment. Utilizing cancer and irAEs-affected skin tissues, they identified identical $\mathrm{T}$ cell receptor (TCR) sequences in both the primary tumors and the affected skin samples, suggesting that the same $\mathrm{T}$ cell clones that recognized tumor antigens also infiltrated the inflamed skin. Using in silico prediction algorithm they projected nine MHC-T cell antigens that were shared between tumor tissue and affected skin. Notably, these antigens were able to stimulate the corresponding antigen specific CD8 and interferon positive $T$ cell clones that were isolated from the peripheral blood of the patients.

These are very exciting findings. irAEs are driven by an inflammatory response, as intrinsically defined by the presence of mononuclear cells infiltrates in the affected 
tissues. However, the nature of the inflammatory responses (e.g., autoinflammatory $v s$. autoimmune) is not clear (6). The present study provides a strong evidence that the mechanism of irAEs is mediated by immune response to auto-antigens. The fact that the irAEs $T$ cell clones responded to self-antigens (distinctively of neo-antigens), should also explain the observation that the same clinical toxicities are observed with different types of cancers. The presence, and the possible contribution, of auto-antibodies to irAEs tissue damages is still under debate.

$\mathrm{T}$ cells recognizing shared lung tumor and skin antigens targeted both organs simultaneously. One explanation is that the autoimmune $\mathrm{T}$ cell clones were primed in the tumor microenvironment and matured in the regional secondary lymphoid tissue, prior to their egress toward the skin where the shared antigens (epitopes) were expressed (7). An alternative explanation is that the autoimmune $\mathrm{T}$ cells emerged from tissue resident or central memory $\mathrm{T}$ cells and that the administration of the anti-PD-1 antibodies resulted in systemic reduced self-tolerance threshold. To categorically support the first explanation, study of unaffected skin $\mathrm{T}$ cells is required, as well as attempting to sort and stimulate peripheral $\mathrm{T}$ cells from patients that failed to respond to anti-PD-1 antibodies and that did not develop skin-related or other sites irAEs. It is also possible that some irAEs were the result of loss of self-tolerance while others were secondary to perturbed regulatory $\mathrm{T}$ cells activity that resulted in loss of tissue specific homeostasis (8). These mechanistic differences may have important implications for future management of specific irAEs.

Despite the broad similarity between NSCLC tissue and skin proteins, some of those identified (such as HSP27 and peroxiredoxin 2) are widely expressed and are not unique to either the lung or the skin. Since the candidate antigens were identified in silico, and not via immunopeptidome enrichment approach, additional selfreactive and immunogenic proteins are likely to exist. TCRs were sequenced and validated in two patients; however, the potential translational impact of this work is noteworthy. Does the presence of autoimmune peripheral clones could serve as a biomarker of irAEs onset? Future implications of this work will allow us to better define the molecular changes associated with $\mathrm{T}$ cell repertoire transition from self-tolerant to sensitized memory auto-reactive cells. Ultimately, these studies raise the question of whether the identification of immunogenic tissue antigen may enable more effective immunotherapy while avoiding self-directed immune responses.
Altogether, this is the first study to document $\mathrm{T}$ cell responses to antigens common to NSCLC tissue and skin as a possible explanation of the inflammatory toxic effects of anti-PD-1 inhibitors. The identification of antigens common to skin and lung that are differentially recognized by peripheral CD8 $\mathrm{T}$ cells of patients with skin toxicity provide a stimulus for comparable studies in patients with a range of other autoimmune toxic effects.

\section{Acknowledgments}

Funding: This work was supported by the National Institute of Health (AI125640 to A Mor) and a cancer foundation (ANON CU19-0774 to A Mor).

\section{Footnote}

Provenance and Peer Review: This article is commissioned and reviewed by the Section Editor Wei Xu (Division of Respiratory Disease, Department of Geriatrics, the First Affiliated Hospital of Nanjing Medical University, Nanjing, China).

Conflicts of Interest: Both authors have completed the ICMJE uniform disclosure form (available at http://dx.doi. org/10.21037/tcr.2019.07.43). The authors have no conflicts of interest to declare.

Ethical Statement: The authors are accountable for all aspects of the work in ensuring that questions related to the accuracy or integrity of any part of the work are appropriately investigated and resolved.

Open Access Statement: This is an Open Access article distributed in accordance with the Creative Commons Attribution-NonCommercial-NoDerivs 4.0 International License (CC BY-NC-ND 4.0), which permits the noncommercial replication and distribution of the article with the strict proviso that no changes or edits are made and the original work is properly cited (including links to both the formal publication through the relevant DOI and the license). See: https://creativecommons.org/licenses/by-nc-nd/4.0/.

\section{References}

1. Wu X, Gu Z, Chen Y, et al. Application of PD-1 Blockade in Cancer Immunotherapy. Comput Struct Biotechnol J 2019;17:661-74. 
2. LaFleur MW, Muroyama Y, Drake CG, et al. Inhibitors of the PD-1 Pathway in Tumor Therapy. J Immunol 2018;200:375-83.

3. Sandigursky S, Mor A. Immune-Related Adverse Events in Cancer Patients Treated With Immune Checkpoint Inhibitors. Curr Rheumatol Rep 2018;20:65.

4. Pauken KE, Dougan M, Rose NR, et al. Adverse Events Following Cancer Immunotherapy: Obstacles and Opportunities. Trends Immunol 2019;40:511-23.

5. Berner F, Bomze D, Diem S, et al. Association of Checkpoint Inhibitor-Induced Toxic Effects With Shared Cancer and Tissue Antigens in Non-Small Cell Lung

Cite this article as: Lerrer S, Mor A. Immune checkpoint inhibitors and the shared epitope theory: from hypothesis to practice. Transl Cancer Res 2019;8(Suppl 6):S625-S627. doi: $10.21037 /$ tcr.2019.07.43
Cancer. JAMA Oncol 2019. [Epub ahead of print].

6. Khan Z, Hammer C, Guardino E, et al. Mechanisms of immune-related adverse events associated with immune checkpoint blockade: using germline genetics to develop a personalized approach. Genome Med 2019;11:39.

7. Sioud M. T-cell cross-reactivity may explain the large variation in how cancer patients respond to checkpoint inhibitors. Scand J Immunol 2018. doi: 10.1111/sji.12643.

8. Davar D, Kirkwood JM. PD-1 Immune Checkpoint Inhibitors and Immune-Related Adverse Events: Understanding the Upside of the Downside of Checkpoint Blockade. JAMA Oncol 2019;5:942-943. 